\title{
Micro-computed tomography assisted distal femur metaphyseal blunt punch compression for determining trabecular bone strength in mice
}

\author{
Uma Sankar ${ }^{\mathrm{a}, \mathrm{b}, \mathrm{c}, \star}$, Zachary J. Pritchard ${ }^{\mathrm{b}}$, Michael J. Voor ${ }^{\mathrm{de}, \mathrm{e}, \star}$
}

aDepartment of Anatomy and Cell Biology, Indiana University School of Medicine, Indianapolis, IN, USA; 'Department of Pharmacology and Toxicology, ' James Graham Brown Cancer Center and Owensboro Cancer Research Program, University of

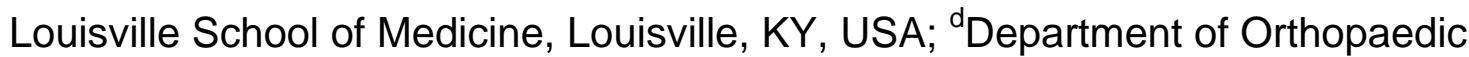
Surgery, University of Louisville School of Medicine, Louisville, KY; ${ }^{\text {DDepartment of }}$ Bioengineering, University of Louisville Speed School of Engineering, Louisville, KY, USA.

*Address correspondences to: Uma Sankar, Ph.D., Department of Anatomy and Cell Biology, Indiana University Purdue University Indianapolis, 635 Barnhill Drive, MS-5055, Indianapolis, IN 46202 USA. Tel: (317) 274-7870, Fax: (317) 856-5710; Email: usankar@iupui.edu; or Michael J. Voor, Ph.D., Orthopaedic Bioengineering Laboratory, University of Louisville, 40292 USA. Tel: (502) 852-7067, Fax: (502) 852-7227, Email: michael.voor@louisville.edu.

Keywords: Metaphyseal cancellous bone strength, distal femur, blunt punch compression, micro-CT, mouse models.

Word Count: 1965 


\section{Abstract}

Shorter generation time and the power of genetic manipulation make mice an ideal model system to study bone biology as well as bone diseases. However their small size presents a challenge to perform strength measurements, particularly of the weightbearing cancellous bone in the murine long bones. We recently developed an improved method to measure the axial compressive strength of the cancellous bone in the distal femur metaphysis in mice. Transverse micro-computed tomography image slices that are $7 \mu \mathrm{m}$ thick were used to locate the position where the epiphysis-metaphysis transition occurs. This enabled the removal of the distal femur epiphysis at the exact transition point exposing the full extent of metaphyseal trabecular bone, allowing more accurate and consistent measurement of its strength. When applied to a murine model system consisting of five month old male wild-type (WT) and $\mathrm{Ca}^{2+} /$ calmodulin dependent protein kinase kinase 2 (CaMKK2) knockout (KO) Camkk2 ${ }^{-/}$mice that possess recorded differences in trabecular bone volume, data collected using this method showed good correlation between bone volume fraction and strength of trabecular bone. In combination with micro-computed tomography and histology, this method will provide a comprehensive and consistent assessment of the microarchitecture and tissue strength of the cancellous bone in murine mouse models. 


\section{Introduction}

With the popularity of mice as models for the study of bone related disease and treatment, biomechanical test methods must be developed to measure the strength of mouse cancellous and cortical bone. It is also desirable to measure the properties of bone from various anatomic sites such as the spine, skull and long bones. Due to their small size, mice present a difficult problem for mechanical testing using traditional test methods. Whereas three-point bending can still be used to study cortical bone in mouse femurs, methods such as direct compression that are commonly used to measure cancellous bone mechanical properties are either less effective or are restricted to the spine (Turner, 2006; Turner and Burr, 1993).

Cancellous bone is of particular interest in the study of bone diseases such as osteoporosis. Therefore better test methods specific to cancellous bone are needed. Micro, nano, and reference-point indentation tests can be used on both cortical and cancellous bone, but those methods measure tissue level properties (Diez-Perez et al., 2010; Turner et al., 1999; Zysset et al., 1999). Because many conditions affect bone morphology more than bone tissue material properties, it is still useful to measure cancellous bone at the apparent level.

We herein present a test method to more consistently measure the axial compressive strength of metaphyseal cancellous bone of the distal femur, as a modification of previously described techniques (An et al., 1997; Dubrow et al., 2007)). This improved method was intended to complement three-dimensional imaging by micro-computed tomography (micro-CT) and the histological techniques used to document the cancellous bone morphology in the same region. We used a murine 
genetic model with known phenotype differences in distal femoral cancellous

microarchitecture. Whereas 5 month-old WT mice have sparse cancellous bone architecture in the metaphyseal regions, $\mathrm{KO}$ mice of this age have robust cancellous bone in the metaphysis (Cary et al., 2013; Pritchard et al., 2015). It was our hypothesis that including the epiphyseal/metaphyseal transition could better determine the strength differences of the metaphyseal trabecular bone in animals with different developmental cancellous bone growth phenotypes.

\section{Methods}

Mice

Five month old male wild-type C57BL/6 (WT, n=10) and Camkk2 ${ }^{--}(\mathrm{KO}, \mathrm{n}=14)$ mice of the C57BL/6 background were housed under a 12-hr light and dark cycle, with food and water provided ad libitum. All care and procedures were performed according to Institutional Animal Care and Use Committee (IACUC) protocols and in compliance with $\mathrm{NIH}$ guidelines on the use and care of laboratory and experimental animals.

\section{$\underline{\text { Micro-CT }}$}

Imaging was performed using a high resolution CT scanner (Actis HR225-150; BIR, Lincolnshire, IL, USA). Distal femurs were imaged at a nominal isotropic voxel resolution of $7 \mu \mathrm{m}$. An approximate length of $5.25 \mathrm{~mm}$ was scanned starting from the distal-most aspect of the condyles and progressing proximally to include the entire metaphysis and some diaphysis. Transverse images were processed using both twodimensional (ImageJ, NIH Image, Bethesda, MD) and three-dimensional software (VG 
Studio Max, Volume Graphics, Heidelberg, DE) (Figure 1). A previously described technique (Cary et al., 2013) was used to determine the metaphyseal trabecular bone volume fraction $(\mathrm{BV} / \mathrm{TV})$.

\section{Epiphysis removal}

For the first group of WT ( $n=5)$ and KO $(n=9)$ femurs tested, a constant length from the distal surface of the femoral condyles was used to cut each femur to expose the cancellous bone of the metaphysis for mechanical blunt punch compression testing. This length was determined by averaging the approximate distance to the growth plate from the micro-CT images for the entire group in each genotype and adding 350 microns to ensure each cut would be proximal to the physis and within the metaphysis. This resulted in trim lengths of approximately $1.60 \mathrm{~mm}(\mathrm{WT})$ and $1.53 \mathrm{~mm}(\mathrm{KO})$ (Table 1). Exact measurements for this first group of femurs were not recorded as they were for the second group.

The reason for not using a more statistically powerful contralateral paired design is because the improved trimming method was developed after the tests on the first 5 WT and $9 \mathrm{KO}$ mice revealed little correlation between mechanical testing and micro-CT determined BV/TV. Thus the Group 1 animals were no longer available when Group 2 testing was done.

For the second group of WT $(n=5)$ and $K O(n=5)$ femurs tested, transverse micro-CT image slices (each $7 \mu \mathrm{m}$ thick) were used to locate the transition between the proximal aspect of the physis and the distal extent of the metaphysis (Table 1). By counting image slices, the distance to the nearest $7 \mu \mathrm{m}$ from the distal end of the condyle to the transition was determined (Figure 1). The epiphysis/metaphysis junction, 
appearing as a cross with four chambers on the micro-CT image (slice 177, Figure 1), represents the region where the metaphyseal trabecular bone begins.

To prepare for removal of the bone distal to the predetermined cutting location, the entire distal end portion of each femur was mounted using a two part epoxy mix (Loctite ${ }^{\circledR}$ Epoxy Quick Set ${ }^{\mathrm{TM}}$, Westlake, OH, USA) atop a socket headed \#10 cap screw (Fastenal, USA) (Figure 2A). Care was taken to be sure the femoral axis was aligned with the axis of the screw. Samples were allowed to cure for 24 hours while kept hydrated and refrigerated.

The femur was then cut to remove the epiphysis using a diamond sectioning saw (Isomet ${ }^{\circledR}$, Lake Bluff, IL USA). The mounted femur was positioned on the micrometer arm of the diamond saw and advanced until the distal part of the condyle first contacted the blade. From this point, the micrometer was used to advance the femur to the calculated epiphysis position for cutting. The femur was kept wet with isotonic saline during the cutting process. The cut surface was perpendicular to the axis of the mounted femur.

\section{Blunt Punch Compression}

Immediately following the removal of the distal ephiphysis, the femur was centered on a servo-hydraulic load frame (Model 858 Bionix, MTS Corp., Eden Prairie, MN). Compression of distal femoral trabecular bone was performed via a blunt (flattipped) cylindrical punch measuring $3 \mathrm{~mm}$ in length and $1 \mathrm{~mm}$ in diameter (Figure 2B) at a loading rate of $1 \mathrm{~mm} / \mathrm{min}$. Due to the curvature/slope of the posterior femur cortex (Figure 1), it was possible for the punch to contact cortical bone after penetrating 
beyond $1.5 \mathrm{~mm}$ during testing. Therefore, alignment of the punch was carefully performed by placing it slightly anterior of dead center to avoid the posterior slope.

No more than the first $1.5 \mathrm{~mm}$ of punch penetration during the test was used to determine strength (Figure 2C). Anything further than that was disregarded as it could involve contact with the cortical wall and give an artificially high force reading.

Confirmation of proper indentation of individual femurs was performed by follow-up micro-CT (Figure 2D). The maximum amount of compressive force measured was divided by the cross-sectional area of the punch tip to determine the apparent strength of the trabecular bone.

$\underline{\text { Statistical analysis }}$

All data are represented as average values \pm standard deviation. Statistical comparisons between groups were made by Student's t-test. Differences with $p$-value $<$ 0.05 were deemed significant. Correlations between BV/TV and trabecular bone strength were determined by linear best-fit with statistical significance of the correlation coefficient at the $p<0.05$ level.

\section{Results}

When the trimming of the distal femur was not controlled by prior imaging (Group 1), the femurs from WT and KO mice showed a significant difference in trabecular microarchitecture (BV/TV) as measured by micro-CT $(p<0.05)$, but there was no difference in measured blunt punch compressive strength (Figure 3A). However, when the trimming of the distal femur was controlled by prior imaging to include similar 
anatomy between individual bones (Group 2), there was a statistically significant difference in measured blunt punch compressive strength (Figure $3 A)(p<0.05)$. The Group 2 WT and KO femurs again demonstrated significant differences in volume fraction $(\mathrm{BV} / \mathrm{TV})$ as measured by micro-CT $(\mathrm{p}<0.05)$. The same data also reveal that the strength values were significantly correlated with the trabecular architecture (BV/TV) only when the controlled trimming method was used (Figures 3B and C).

\section{Discussion}

Bone provides structure for locomotion and protection for vital organs. Without adequate bone strength and fracture resistance, the skeleton is compromised. Thus, any measure of bone quality should be able to directly or indirectly assess bone strength. High resolution imaging studies are particularly useful for assessing bone because they can assess bone three-dimensionally and nondestructively. In the case of trabecular bone of known or consistent tissue quality, the three-dimensional microarchitecture of the trabeculae should be strongly correlated to the strength of the trabecular bone. If, on the other hand, the tissue level quality of the bone varies, imaging studies alone may not be adequate to assess bone quality.

This study was intended to provide a companion mechanical test to micro-CT assessment of trabecular bone in the mouse models used for studying bone phenotypes. Because the spatial distribution of trabecular bone in the metaphyseal region goes through a transition from very low density at the diaphyseal end to its maximum density near the physis, it is difficult to consistently test the strength of the trabecular bone at the apparent level. Apparent level mechanical testing must involve 
multiple trabeculae in order to reflect the combined effects of both bone architecture and tissue properties. Our data (Figure 3) illustrate the importance of including the full extent of the metaphyseal trabecular structure in any mechanical assessment of strength. These data further support the strong correlation between architecture and strength of trabecular bone when the tissue properties are similar across the range of bone volume fractions tested. The similarity of bone tissue properties was previously established for the WT and KO $\left(\right.$ Camkk2 $\left.^{-/}\right)$mice used in the present study (Pritchard et al., 2015).

There was a large difference in the strength measured between the two trimming methods. The samples trimmed to the epiphysis/metaphysis transition point through micro-CT based individual measurements displayed higher trabecular strength compared to samples trimmed within the metaphysis, using a constant average distance. This reflects the contribution of the connectedness of the trabeculae when a thin portion of the physis at the transition is included in the test sample. In this way the trabecular architecture of the metaphyseal bone is functioning effectively to carry the axial load transmitted from the joint surface through the epiphysis to the diaphysis.

The consistency of our improved trimming method is reflected in the smaller variance (calculated as Coefficient of Variation) in measured stiffness, yield, and ultimate strength compared to the averaged distance trimming method (Figure 3) or the epiphyseal grinding method used by Dubrow and colleagues (Table 2) (Dubrow et al., 2007). Our method could potentially be improved by systematically varying the trim depth to determine the precise level that provides the smallest variance in strength, but such a follow up study would require a very large number of mice. 
By providing a consistent and convenient mechanical test method to assess the apparent level trabecular bone strength, this technique can be used in the future to determine when there are differences in tissue-level quality of the trabecular bone. For example, if the bone volume fraction measurements from two different groups of mice are similar, but there are significant differences in the blunt punch compressive strengths between groups after controlled trimming of the metaphysis, then one may conclude that the difference in strength is attributable to the tissue quality rather than the architecture. This would support further investigation by another tissue level test method such as micro or nano-indentation. Additionally, this method of consistent trimming at the metaphyseal/epiphyseal junction would allow similar blunt punch testing to be done in the mouse proximal tibia, where there is often so little metaphyseal trabecular bone tissue that it is difficult to perform mechanical tests. Finally, this method could be scaled up and translated to the larger rat model for testing of the femur or tibia.

\section{Acknowledgements}

This work was supported in part by grants from the American Cancer Society (RSG 13301-01), Department of Defense/CDMRP (PR121604), National Institutes of Health (NIAMS R01AR068332) and an intramural competitive enhancement grant from the Office of the Executive Vice President for Research at the University of Louisville.

\section{Conflict of Interest Statement:}


No authors have any relationships due to funding, consultancy, stock ownership, or any other relationships that have the potential to bias their work as it relates to the subject of this manuscript.

\section{References}

An, Y.H., Zhang, J., Kang, Q., and Friedman, R.J. (1997). Mechanical properties of rat epiphyseal cancellous bones studied by indentation testing. Journal of materials science Materials in medicine 8, 493-495.

Cary, R.L., Waddell, S., Racioppi, L., Long, F., Novack, D.V., Voor, M.J., and Sankar, U. (2013). Inhibition of $\mathrm{ca}(2+)$ /calmodulin-dependent protein kinase kinase 2 stimulates osteoblast formation and inhibits osteoclast differentiation. Journal of bone and mineral research : the official journal of the American Society for Bone and Mineral Research $28,1599-1610$.

Diez-Perez, A., Guerri, R., Nogues, X., Caceres, E., Pena, M.J., Mellibovsky, L., Randall, C., Bridges, D., Weaver, J.C., Proctor, A., et al. (2010). Microindentation for in vivo measurement of bone tissue mechanical properties in humans. Journal of bone and mineral research : the official journal of the American Society for Bone and Mineral Research 25, 1877-1885.

Dubrow, S.A., Hruby, P.M., and Akhter, M.P. (2007). Gender specific LRP5 influences on trabecular bone structure and strength. J Musculoskelet Neuronal Interact 7, 166173.

Pritchard, Z.J., Cary, R.L., Yang, C., Novack, D.V., Voor, M.J., and Sankar, U. (2015). Inhibition of CaMKK2 reverses age-associated decline in bone mass. Bone 75, 120127.

Turner, C.H. (2006). Bone strength: current concepts. Annals of the New York Academy of Sciences 1068, 429-446.

Turner, C.H., and Burr, D.B. (1993). Basic biomechanical measurements of bone: a tutorial. Bone 14, 595-608.

Turner, C.H., Rho, J., Takano, Y., Tsui, T.Y., and Pharr, G.M. (1999). The elastic properties of trabecular and cortical bone tissues are similar: results from two microscopic measurement techniques. Journal of biomechanics 32, 437-441. 
Zysset, P.K., Guo, X.E., Hoffler, C.E., Moore, K.E., and Goldstein, S.A. (1999). Elastic modulus and hardness of cortical and trabecular bone lamellae measured by nanoindentation in the human femur. Journal of biomechanics 32, 1005-1012.

\section{Figure Legends:}

Figure 1. Representative samples from Groups 1 and 2 to demonstrate the method for determining the cutting length for the removal of distal epiphysis to expose the metaphyseal cancellous bone in mouse femurs. Group 1: Trim depth was determined by averaging the approximate distance to the physis for the entire group in each genotype and adding 350 microns to ensure each cut would be proximal to the physis and within the metaphyseal cancellous bone. Group 2: The exact distance from the distal condyle to the epiphysis/metaphysis junction for each femur was calculated by counting the number of micro-CT image slices (each $7 \mu \mathrm{m}$ thick). This cutting distance was utilized to trim the distal femurs to expose the metaphyseal cancellous bone.

Figure 2. Blunt punch compression of the metaphyseal cancellous bone in mouse femur. (A) Distal femur mounted using a two part epoxy mix on top of a socket headed \#10 cap screw. (B) After removal of the epiphysis the distal femur is centered on a servo-hydraulic frame for compression using a flat-tipped cylindrical punch measuring 3 $\mathrm{mm}$ in length and $1 \mathrm{~mm}$ in diameter at a loading rate of $1 \mathrm{~mm} / \mathrm{min}$. (C) Graph displaying force $(\mathrm{N})$ versus punch penetration depth $(\mathrm{mm})$ during punch compression strength of the trabecular bone. Only the first $1.5 \mathrm{~mm}$ of punch penetration depth was 
used to determine trabecular bone strength. (D) Micro-CT cross-sectional images before and after punch compression.

Figure 3. Application of the improved method to determine trabecular bone strength. (A) Average BV/TV (\%) and ultimate strength (MPa) measurements from WT and KO femur samples when the trimming of the distal femurs were performed based on a constant average distance value (Group 1) or based on micro-CT aided individual distance from condyle to the epiphysis/metaphysis transition point (Group 2). Yield stress (MPa) and stiffness $(\mathrm{N} / \mathrm{mm})$ measurements from the indicated cohorts are also shown. (B) Plot showing no correlation between trabecular bone volume and compression strength when trimming of distal femur was not controlled by prior microCT imaging. (C) Graph showing that applying micro-CT imaging-based information to trim epiphysis in individual mice results in a statistically significant $(p<0.05)$ correlation between trabecular bone volume and compression strength.

Table 1. Trim Lengths of the Distal Femurs from each Group

\begin{tabular}{|c|c|c|c|}
\hline \multicolumn{2}{|c|}{ Group 1} & \multicolumn{2}{|c|}{ Group 2} \\
\hline WT & $\mathrm{KO}\left(\right.$ Camkk2 $\left.{ }^{-/-}\right)$ & WT & $\mathrm{KO}\left(\right.$ Camkk2 $\left.^{-/-}\right)$ \\
\hline & & 1.211 & 1.120 \\
\hline & & 1.274 & 1.155 \\
\hline & & 1.239 & 1.155 \\
\hline & & 1.239 & 1.323 \\
\hline & & 1.274 & 1.211 \\
\hline $1.60^{*}$ & $1.53^{*}$ & 1.247 & 1.184 \\
\hline
\end{tabular}

Average *approximate

Table 2. Coefficients of Variation for Bone Volume Fraction and Biomechanical Test Measures Present Study 


$\begin{array}{lccc}\begin{array}{c}\text { Trabecular Bone } \\ \text { Measure }\end{array} & \begin{array}{c}\text { Data from Dubrow et al. } \\ (2007)\end{array} & \begin{array}{c}\text { without micro-CT } \\ \text { assisted trimming }\end{array} & \begin{array}{c}\text { with micro-CT } \\ \text { assisted trimming }\end{array} \\ \text { BV/TV } & 0.26 & 0.18 & 0.13 \\ \text { Stiffness } & 0.64 & 0.80 & 0.44 \\ \text { Yield Strength } & 0.72 & 0.54 & 0.26 \\ \text { Ultimate Strength } & 0.61 & 0.28 & 0.22\end{array}$




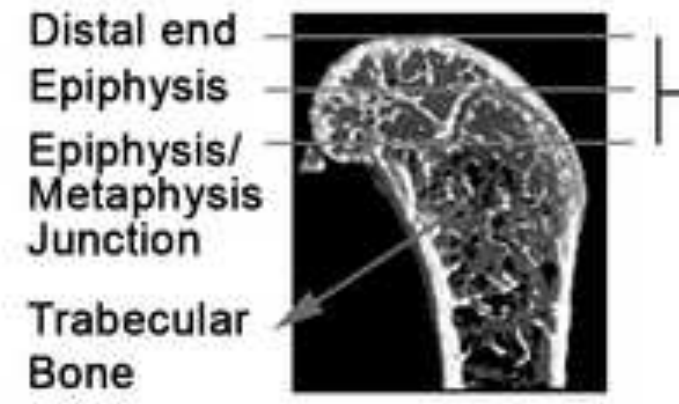

Group 1

Cut location $=$ Slice 227

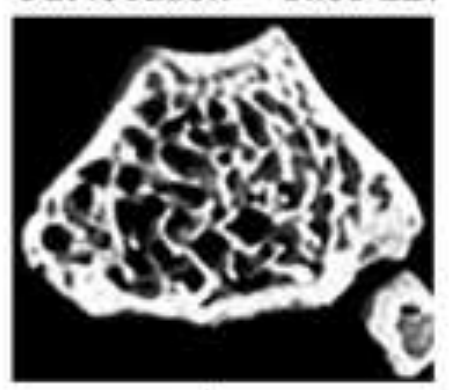

1

Trim depth using average distance to physis plus $350 \mu \mathrm{m}$

Approximate cut length $=1.53 \mathrm{~mm}$

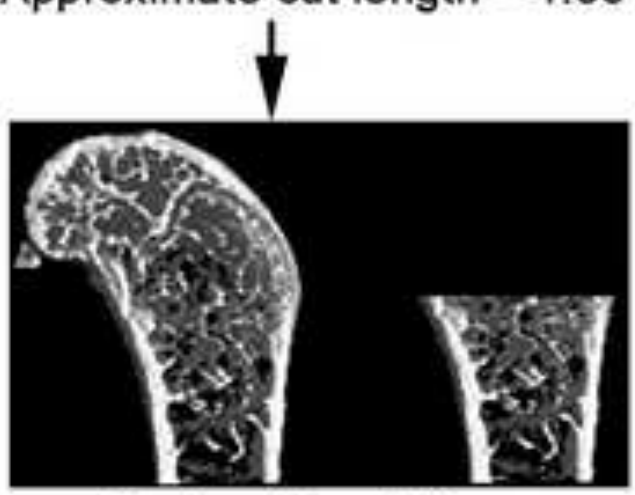

Post cutting $1.53 \mathrm{~mm}$
Top-down micro-CT image

cross-

sections ( 7

$\mathrm{mm}$ each) of

epiphysis

\section{Group 2}
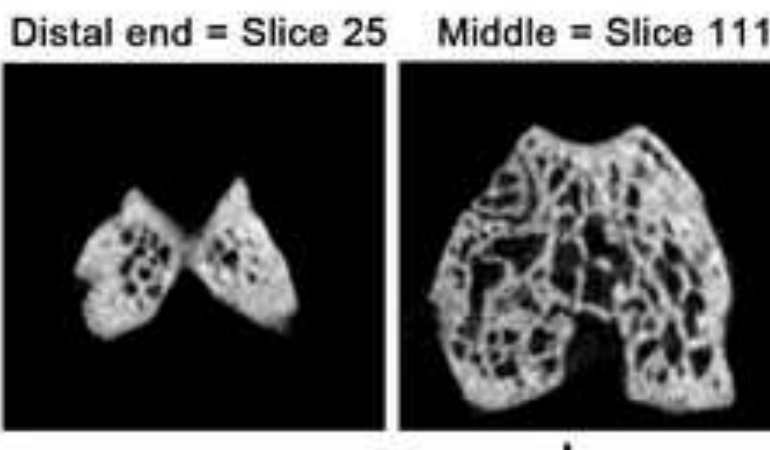

1

Each cross-section or slice $=7 \mu \mathrm{m}$ thickness 177 slices $=7 \times 177=1239 \mu \mathrm{m}=1.24 \mathrm{~mm}$ Cutting length $=1.24 \mathrm{~mm}$

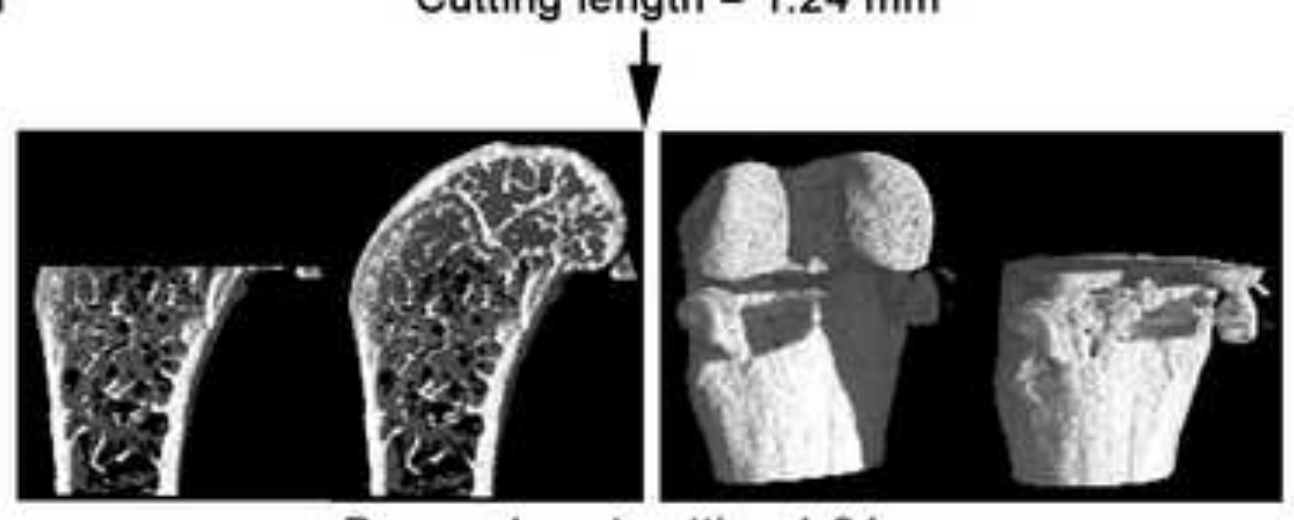

Pre- and post cutting $1.24 \mathrm{~mm}$

Junction $=$ Slice 177

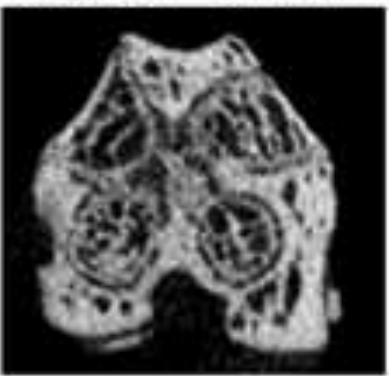

\section{(n)}


A

Epoxy-mounted distal femur

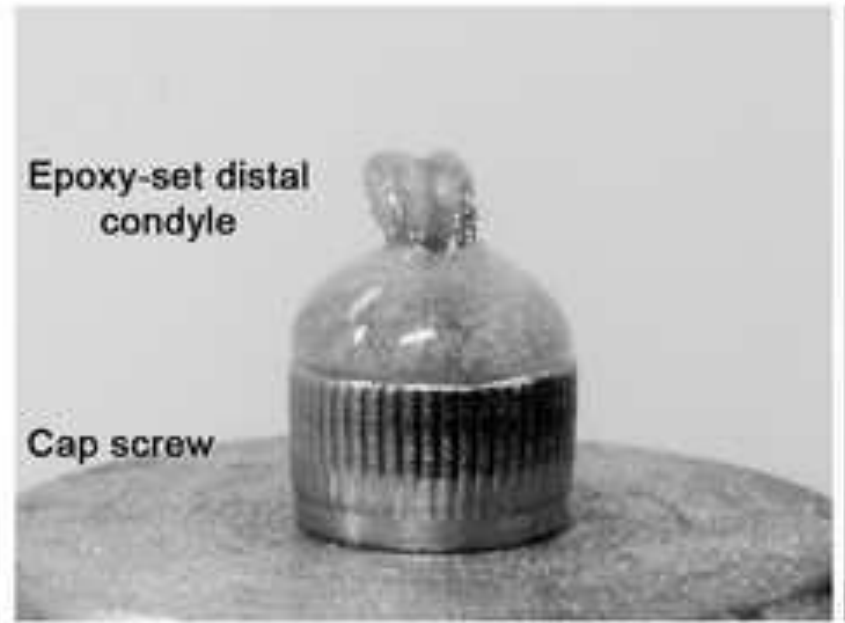

Figure 2

Mounted femur prior to compression

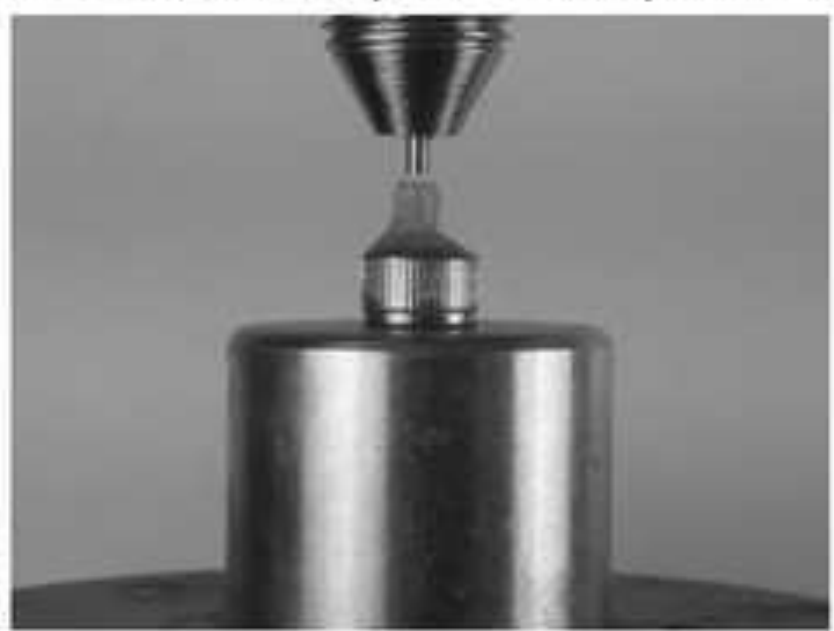

C

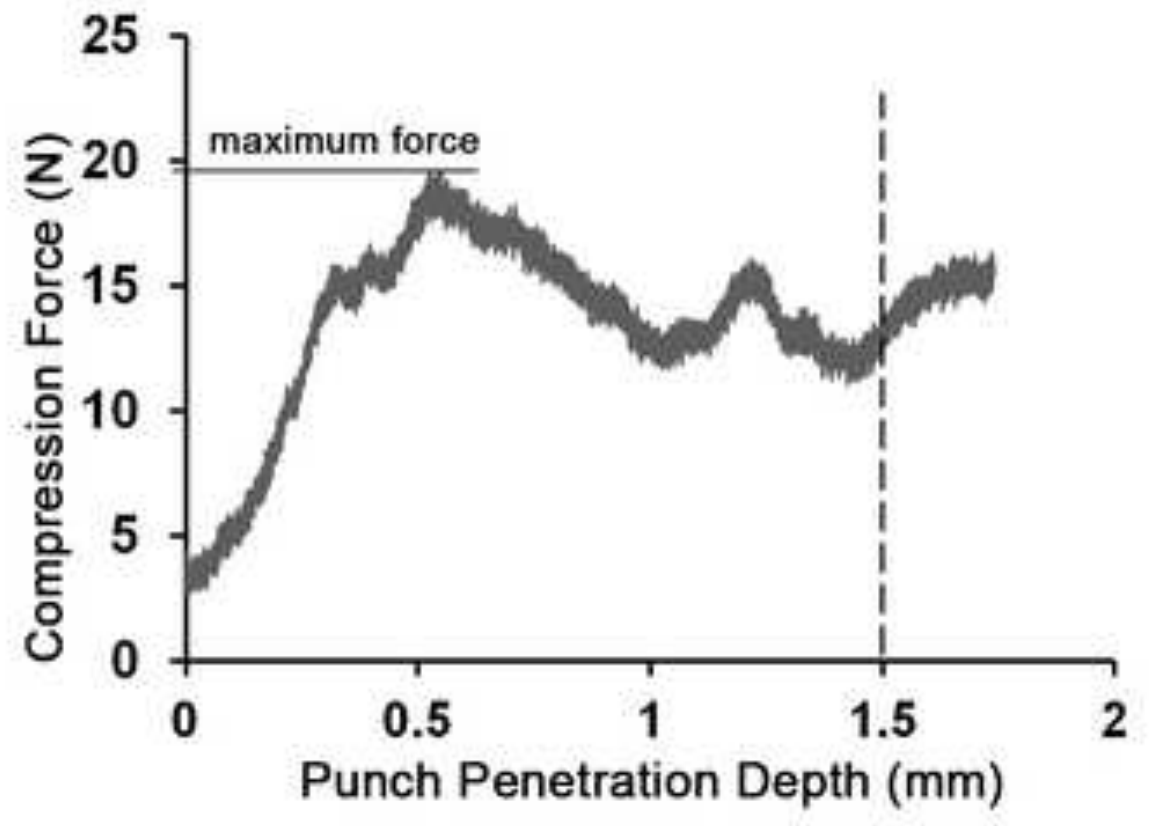

D

Micro-CT crosssections before and after compression

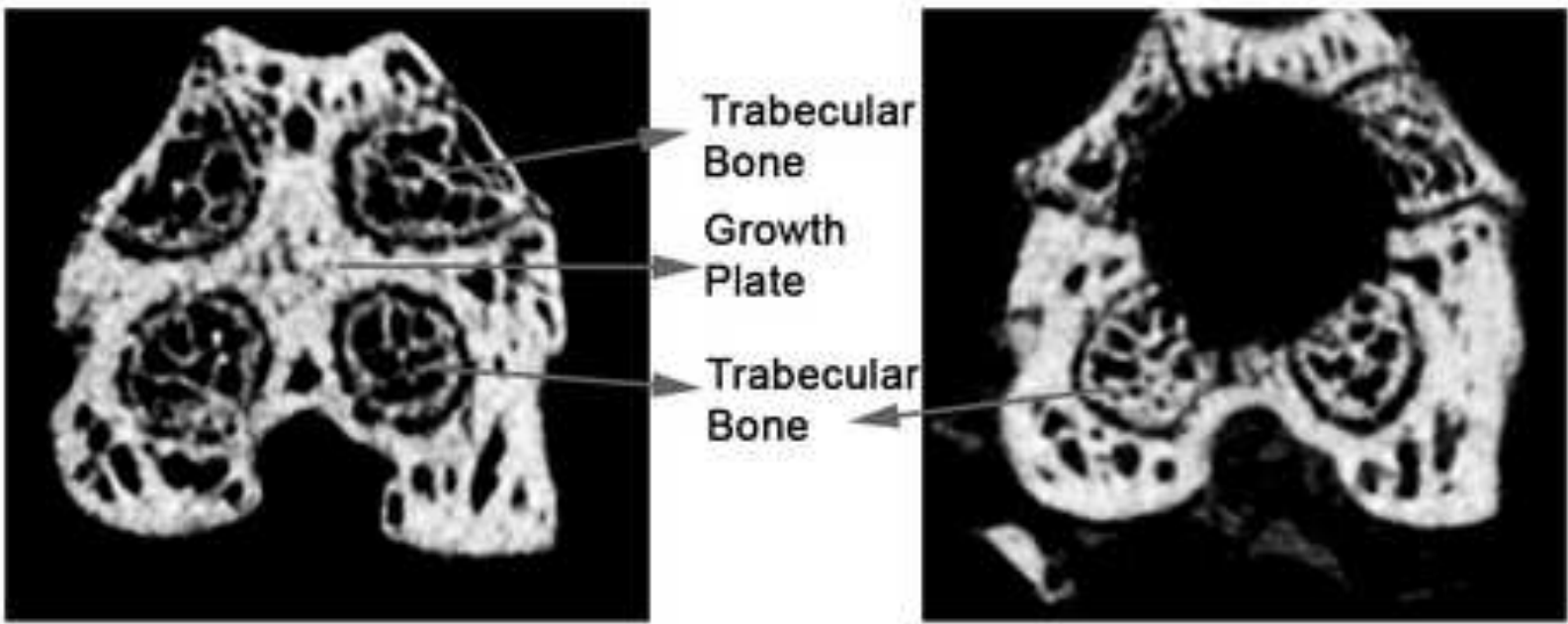




\begin{tabular}{lcccc}
\hline & \multicolumn{2}{c}{ Group 1 } & \multicolumn{2}{c}{ Group 2 } \\
\cline { 2 - 5 } & $\begin{array}{c}\text { Distal femur trimmed at constant } \\
\text { distance from condyle }\end{array}$ & $\begin{array}{c}\text { Distal femur trimmed at variable individual } \\
\text { distance from condyle to transition }\end{array}$ \\
\cline { 2 - 5 } & WT $(n=5)$ & KO $(n=9)$ & WT $(n=5)$ & KO $(n=5)$ \\
\hline BV/TV (\%) & $19.84 \pm 3.9$ & $29.36 \pm 5^{*}$ & $24.34 \pm 2.3$ & $32.97 \pm 5.75^{*}$ \\
Uitimate Strength (MPa) & $16.98 \pm 6.6$ & $17.18 \pm 3$ & $41.42 \pm 8.2$ & $57.57 \pm 14^{*}$ \\
Yield Stress (MPa) & $9.02 \pm 6.3$ & $11.56 \pm 4.3$ & $31.86 \pm 7$ & $41.34 \pm 12.2$ \\
Stiffness (N/mm) & $114 \pm 80$ & $73.05 \pm 65$ & $194.9 \pm 62$ & $202 \pm 113.1$ \\
\hline
\end{tabular}

Average values $*$ standard deviation are presented

$\because p<0.05$ when compared to WT
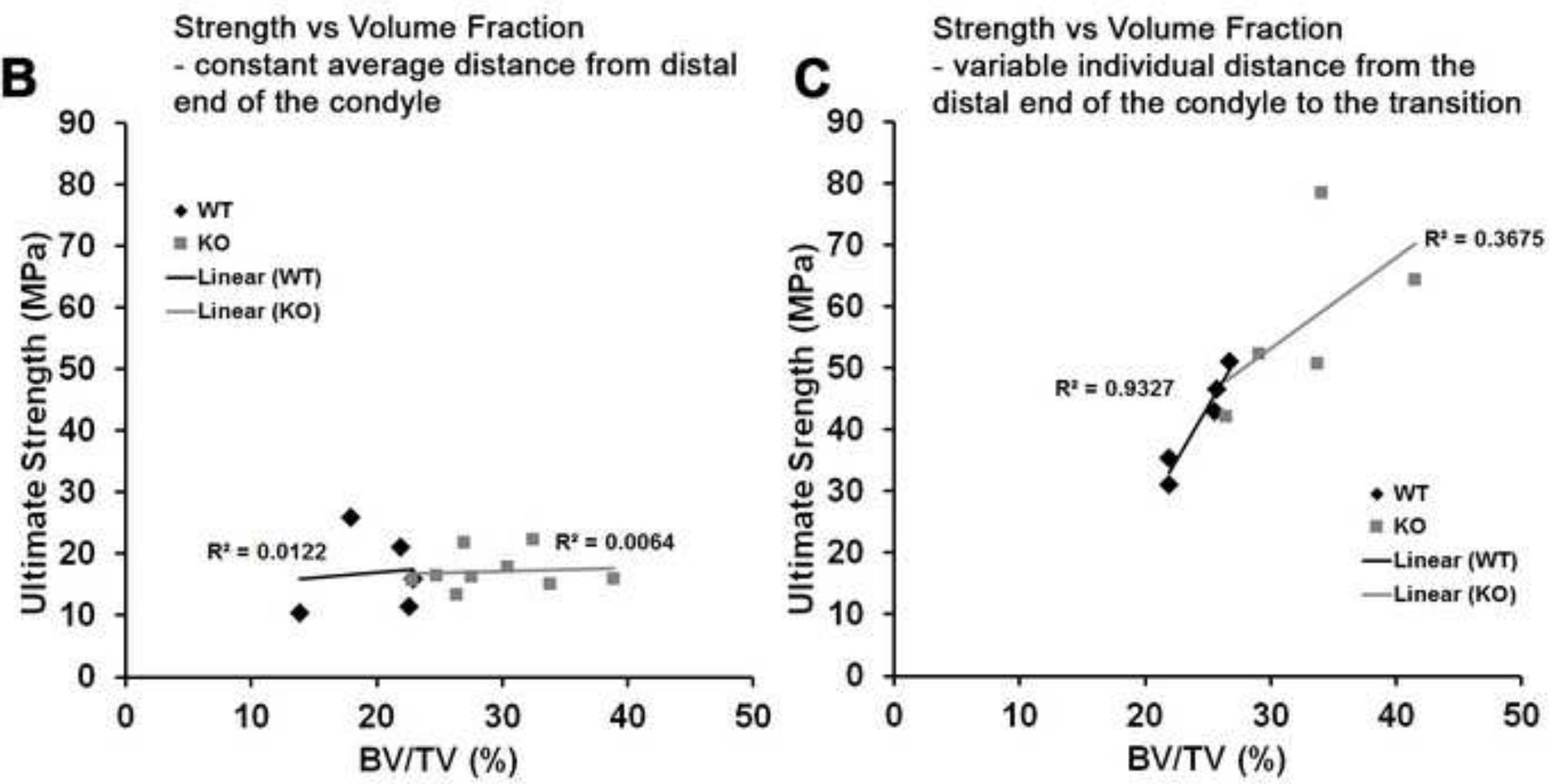
Table 1. Trim Lengths of the Distal Femurs from each Group

\begin{tabular}{|c|c|c|c|c|}
\hline & & & & \\
\hline & WT & $\mathrm{KO}\left(\right.$ Camkk2 $\left.{ }^{-/-}\right)$ & WT & $\mathrm{KO}\left(\right.$ Camkk2 $\left.2^{--}\right)$ \\
\hline & & & 1.211 & 1.120 \\
\hline & & & 1.274 & 1.155 \\
\hline & & & 1.239 & 1.155 \\
\hline & & & 1.239 & 1.323 \\
\hline & & & 1.274 & 1.211 \\
\hline $\begin{array}{l}\text { Average } \\
\text { *approximate }\end{array}$ & $1.60^{*}$ & $1.53^{*}$ & 1.247 & 1.184 \\
\hline
\end{tabular}


Table 2. Coefficients of Variation for Bone Volume Fraction and Biomechanical Test Measures

\begin{tabular}{lccc} 
Trabecular Bone & Data from Dubrow et al. & \multicolumn{2}{c}{ Present Study } \\
\cline { 3 - 4 } Measure & $(2007)$ & without micro-CT & $\begin{array}{c}\text { with micro-CT } \\
\text { assisted trimming }\end{array}$ \\
BV/TV & 0.26 & 0.18 & 0.13 \\
Stiffness & 0.64 & 0.80 & 0.44 \\
Yield Strength & 0.72 & 0.54 & 0.26 \\
Ultimate Strength & 0.61 & 0.28 & 0.22
\end{tabular}

